\title{
Membrane Computing Method for Power System Dynamic Economic Dispatch Optimization Problem
}

\author{
Min XIE ${ }^{\mathrm{a}}$, Yuxin DU ${ }^{\mathrm{b}, *}$, Peijun CHENG ${ }^{\mathrm{c}}$, Xintong HU' ${ }^{\mathrm{d}}$, Mingbo LIU $^{\mathrm{e}}$ \\ School of Electric Power, South China University of Technology, Wushan Road, Guangzhou \\ 510640, China \\ aminxie@scut.edu.cn, binzi6726@163.com, ${ }^{\mathrm{c}} 377839433 @ q q . c o m$, \\ d894685795@qq.com, epmbliu@scut.edu.cn \\ *corresponding author
}

Keywords: Membrane Computing, Power System, Dynamic Economic Dispatch

\begin{abstract}
The membrane computing method is proposed to solve the power system dynamic economic dispatch problem in this paper. The unit generation power during 24 hours is taken as the membrane object. Based on the cell type nested membrane structure, a set of evolution rules including crossover, mutation, correction, reserve, transition, abstraction and target indication are established to optimize the dynamic economic dispatch of power system. The IEEE 39-bus system is used as the test example. The simulation results show that the optimization problem of dynamic economic dispatch can be solved effectively by the proposed membrane computing method, which can obtain a better optimal solution and has a more stable results comparing with the genetic algorithm and particle swarm algorithm. This proposed membrane computing method has a good application prospect in optimization.
\end{abstract}

\section{Introduction}

Membrane computing is a heuristic natural algorithm ${ }^{[1]}$ proposed in 1998 by Gheorghe Păun, a member of the European and the Romanian academy of sciences, and has become a new research field in computer science. The model based on membrane computing is called membrane system, or $P$ system $^{[2]}$. In recent years, optimization problems such as TSP, DNA sequence design, knapsack problem, robot path planning, and power system reconstruction are successfully solved by membrane computing method. Meanwhile, membrane computing method is also involved in the research fields such as optimization, fault diagnosis, systems and synthetic biology, mobile robot control, economics and computer graphics ${ }^{[3]}$.

Economic dispatch problem is one of the typical power system optimization problems, which is a high-dimensional, nonlinear and multi constrained in mathematic view ${ }^{[4]}$. Traditional mathematical algorithms such as interior point method, approximate dynamic programming ${ }^{[5]}$ and Lagrange relaxation method ${ }^{[6]}$, and some intelligent optimization methods such as genetic algorithm ${ }^{[7]}$, 
particle swarm algorithm ${ }^{[9]}$ are used to solve the dynamic economic dispatch problem. Membrane computing method is used in this paper, based on the cell type nested membrane structure, a set of evolution rules including crossover, mutation, correction, reserve, transition, abstraction and target indication are established to optimize the dynamic economic dispatch of power system. The validity of the proposed method is verified and tested on the IEEE-39 bus system.

\section{The Mathematical Model for Power System Dynamic Economic Dispatch Optimization Problem}

\subsection{Objective Function}

The minimum total fuel consumption is taken as the optimization goal of the economic dispatch problem, which generally expressed as quadratic function as follow:

$$
\min f=\sum_{t=1}^{24} \sum_{i=1}^{N g}\left(A_{i, 2} P_{i, t}^{2}+A_{i, 1} P_{i, t}+A_{i, 0}\right)
$$

Where $\mathrm{Ng}$ is the amount of generators; $P_{i, t}$ is generated power of unit $i$ at $t$ th hour; $A_{i, 2}, A_{i, 1}, A_{i, 0}$ are unit cost coefficients.

\subsection{Constraint Conditions}

The constraints of the economic dispatch problem are divided into system constraints and unit features constraints, including power balance constraints, unit output constraints, ramping rate constraints and transmission power constraints.

Power balance constrains:

$$
\sum_{i=1}^{N g} P_{i, t}=P_{L o a d, t}
$$

where $P_{\text {Load, } t}$ is the load at $t$ th hour.

Unit output constraints:

$$
P_{i \min } \leq P_{i} \leq P_{i \max }
$$

where $P_{\text {imax }}$ and $P_{\text {imin }}$ are the maximum and minimum generation limit of unit $i$.

Ramping rate constraints:

$$
\left\{\begin{array}{l}
P_{i, t}-P_{i, t-1} \leq r_{u i} T \\
P_{i, t-1}-P_{i, t} \leq r_{d i} T
\end{array}\right.
$$

where $r_{d i}$ and $r_{u i}$ are the up and down ramp limit of the unit $i$.

Transmission power constraints:

$$
-P_{i j \max } \leq P_{i j} \leq P_{i j \max }
$$

where $P_{i j}$ is the real branch transmission power; $P_{i j m a x}$ is the branch transmission power limit.

\subsection{Unconstrained Dynamic Economic Dispatch Optimal Model Based on Penalty Function}

As mentioned above, the dynamic economic dispatch model is a typical multiple constrained nonlinear optimization problem. To facilitate the application of the membrane computing method, 
quadratic penalty function is used to deal with the constraints, transforming the dynamic economic dispatch problem into unconstrained problem.

For convenient expression, the economic dispatch model shown in formula (1)-(5) is abbreviated to the general formula (6).

$$
\begin{array}{ll}
\min & f(\boldsymbol{x}) \\
\text { s.t. } & g_{i}(\boldsymbol{x}) \leq 0 \\
& h_{j}(\boldsymbol{x})=0
\end{array}
$$

The model (6) is transformed into an unconstrained optimization model shown in formula (7) by adding quadratic penalty terms, where the quadratic penalty function is a simple penalty function without providing any initial feasible solution.

$$
\min Q(\boldsymbol{x})=f(\boldsymbol{x})+\mu_{1} \sum_{g} \max (\mathrm{g}(\boldsymbol{x}), 0)^{2}+\mu_{2} \sum_{h} \max (|h(\boldsymbol{x})|-\delta, 0)^{2}
$$

Where the penalty factors $\mu_{1}, \mu_{2}>0$, representing the acceptable violation domain while the equality constraints are converted into inequality constraints.

\section{Principle and Evolution Rules of Membrane Computing Method}

In the sight of cytobiology, endomembrane is composed of all membranous organelles related closely in structure and function, including endoplasmic reticulum, golgi apparatus, lysosome, various transport vesicles and nuclear membrane, etc. With the function of processing and transporting intracellular material and protein, biological activities such as transportation and secretion of protein in the cell, processing and synthesis of glycoprotein, hydrolysis of protein, directional transportation of plasmalemmal vesicle, are inseparable from the golgi apparatus ${ }^{[11]}$. Inspired by the important role golgi apparatus playing in living cells, a membrane computing method is proposed to solve economic dispatch problem.

Inspired by cellular activities, the bionic optimization algorithm based on membrane computing simulates the production, transport and communication mechanism of intracellular matter. Membrane structure ${ }^{[12]}$, objective and evolution rule are three basic elements of membrane computing.

In this paper, the unit generation power during 24 hours is taken as the membrane object, the cell type nested membrane structure is shown in Figure 1, where membrane 0 is the surface membrane, membrane 1 to $m$ are parallel basic membrane, membrane $G$ is the golgi membrane.

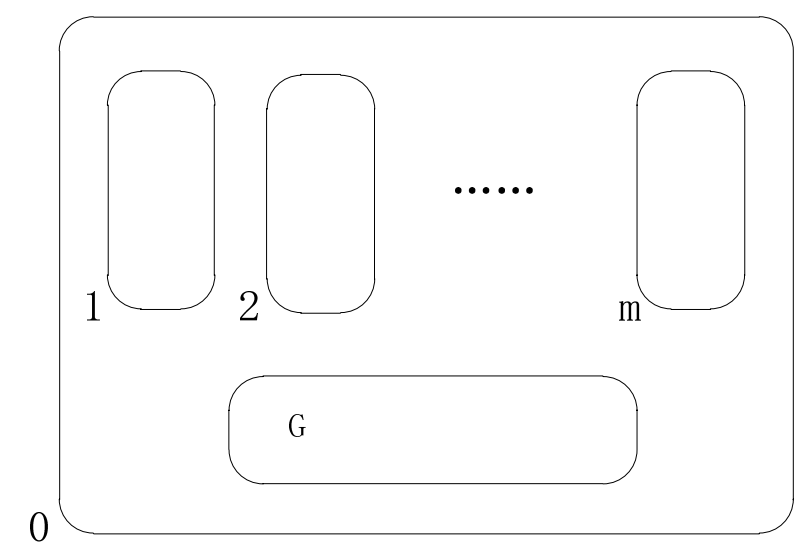

Figure 1 membrane structure drawing. 
The evolution rules include crossover, mutation, correction, reserve, transition, abstraction and target indication rules. Among them, crossover, mutation, correction, reserve rules are executed in the basic membranes, transition, abstraction and target indication rules are executed in golgi membrane after it's activated. The calculation rules are as follows:

(1) Crossover rule

The crossover rule is executed when the setting crossover computational probability is satisfied. First, two adjacent objects are numerically crossed and the original object is replaced by the good new one, then two randomly selected objects crossed in a certain interval and the original object is replaced by the good new one, which can be describe in mathematical formula as follows:

First step:

$$
\left\{\begin{array}{l}
X_{i}^{\prime}=\eta \times X_{i}+(1-\eta) \times X_{i+1} \\
X_{i+1}^{\prime}=(1-\eta) \times X_{i}+\eta \times X_{i+1}
\end{array}\right.
$$

where $X_{i}^{\prime}$ and $X_{i+1}^{\prime}$ are the new objects; $X_{i}$ and $X_{i+1}$ are the original objects; $\eta$ is the random number subjected to uniform distribution in $[0,1]$.

Second step:

$$
\left\{\begin{array}{l}
X_{i}=\left(x_{i 1}, \cdots, x_{i a}, \cdots, x_{i b}, \cdots, x_{i l}\right), X_{k}=\left(x_{k 1}, \cdots, x_{k a}, \cdots, x_{k b}, \cdots, x_{k l}\right) \\
X_{i}^{\prime}=\left(x_{i 1}, \cdots, x_{k a}, \cdots, x_{k b}, \cdots, x_{i l}\right), X_{k}^{\prime}=\left(x_{k 1}, \cdots, x_{i a}, \cdots, x_{i b}, \cdots, x_{k l}\right)
\end{array}\right.
$$

where $a, b \in[1, l] ;[\mathrm{a}, \mathrm{b}]$ is the randomly selected crossover range.

(2) Mutation rule

When the setting mutation computational probability is satisfied, mutation rule is executed, a mutation increment is added to the original object, which can be described in formula as follows.

$$
\left\{\begin{array}{l}
X_{i}^{\prime}=X_{i}+\alpha \\
\alpha=\text { wide } \times r /\left(N_{o} \times N_{c} \times c\right)
\end{array}\right.
$$

where $\alpha$ is the mutation increment; wide is the variable width; $r$ is the random number subjected to the standard normal distribution; No and NCO are the amount of basic membranes and computing cycles; $c$ is the present computing cycle.

(3) Correction rule

The correction rule is executed to modify the elements beyond the scope, especially after the mutation rule.

$$
\left\{\begin{array}{l}
x_{i j}^{\prime}=x_{i j}-x_{i j \max }, x_{i j}>x_{i j \max } \\
x_{i j}^{\prime}=x_{i j \min }-x_{i j}, x_{i j}<x_{i j \min }
\end{array}\right.
$$

where $x_{i j m a x}$ and $x_{i j m i n}$ are the maximum and minimum value of $x_{i j}$.

(4) Reserve rule

The reserve rule is executed after the crossover, mutation and correction rule to improve the efficiency of the algorithm. The sub optimal object set is reserved in the basic membranes while the optimal object set is sent out and the rest objects are removed. The speed and quality of the algorithm can be improved for the sub optimal value interacting with optimal and random values, 
while the reserved objects executing rules along with original objects and communicating objects in next computing cycle.

(5) Transition rule

The transition rule is executed after the golgi membrane activated. First, two randomly selected elements are interchanged if they are in the range of the other one, then the old object is replaced by the new object if the new one is better.

$$
\left\{\begin{array}{l}
X=\left(x_{1}, \cdots, x_{p}, \cdots, x_{q}, \cdots, x_{l}\right) \\
X^{\prime}=\left(x_{1}, \cdots, x_{q}, \cdots, x_{p}, \cdots, x_{l}\right) \\
X_{q \min } \leq x_{p} \leq x_{q \max } \wedge x_{p \min } \leq x_{q} \leq x_{p \max }
\end{array}\right.
$$

(6) Abstraction rule

The abstraction rule is executed after the golgi membrane activated. First, the elements of the optimal object are replaced one by one by the elements from sub optimal object of the same position. Then the element from the sub optimal object is reserved if the new object is better than the old optimal one, or remain the old optimal one.

$$
\left\{\begin{array}{l}
X_{a}=\left(x_{a 1}, \cdots, x_{a j}, \cdots, x_{a l}\right) \\
X_{b}=\left(x_{b 1}, \cdots, x_{b j}, \cdots, x_{b l}\right) \\
X^{\prime}=\left(x_{1}, \cdots, x_{j}, \cdots, x_{l}\right) \quad x_{j} \in\left\{x_{a j}, x_{b j}\right\}
\end{array}\right.
$$

(7) Target indication rule

Direction vector and target vector are calculated whether the golgi membrane is activated or not, while the target object is calculated after the golgi membrane is activated. Direction vector, target vector and target object can be describe as follows.

$$
\left\{\begin{array}{l}
X=\vec{\lambda}+\left(X_{\text {new }}-X_{\text {old }}\right) \\
X_{S 1}=X+\vec{\lambda} \\
X_{S 2}=X-\vec{\lambda}
\end{array}\right.
$$

where $\left(X_{\text {new }}-X_{\text {old }}\right)$ is the direction vector; $\vec{\lambda}$ is the target vector; $X_{S 2}$ and $X_{S 1}$ are the target objects.

\section{Membrane Computing Method Steps of Dynamic Economic Dispatch Problem}

The main steps are summarized as follows:

Step 1: Initialize the parameter settings. Set the amount of membranes and objects (the unit generation power during 24 hours is taken as the object), computational probability of evolution rules and the activating condition of golgi membrane.

Step 2: Start calculation. First, $N_{o}$ initial objects and $N_{c o}$ initial objects are sent to the first basic membrane, No best objects are picked out and crossover, mutation and correction rules work. Then, $N_{c o}$ best objects are picked out as communicating objects and sent to the next basic membrane and golgi membrane. Afterward, $N_{s v}$ next-best objects are remained in this basic membrane and the others are deleted. 
Step 3: If the activating condition of golgi membrane is satisfied, transition, abstraction and target indication rules are executed, then new communicating objects are sent out to the next basic membrane. If not, return to step 2.

Step 4: If this cycle is completed, next cycle start to calculate. If not, return to step 2 and next basic membrane start to calculate.

Step 5: If the last cycle is completed, output the best object. If not, return to step 2.

The flow chart of the proposed method is shown in Figure 2.

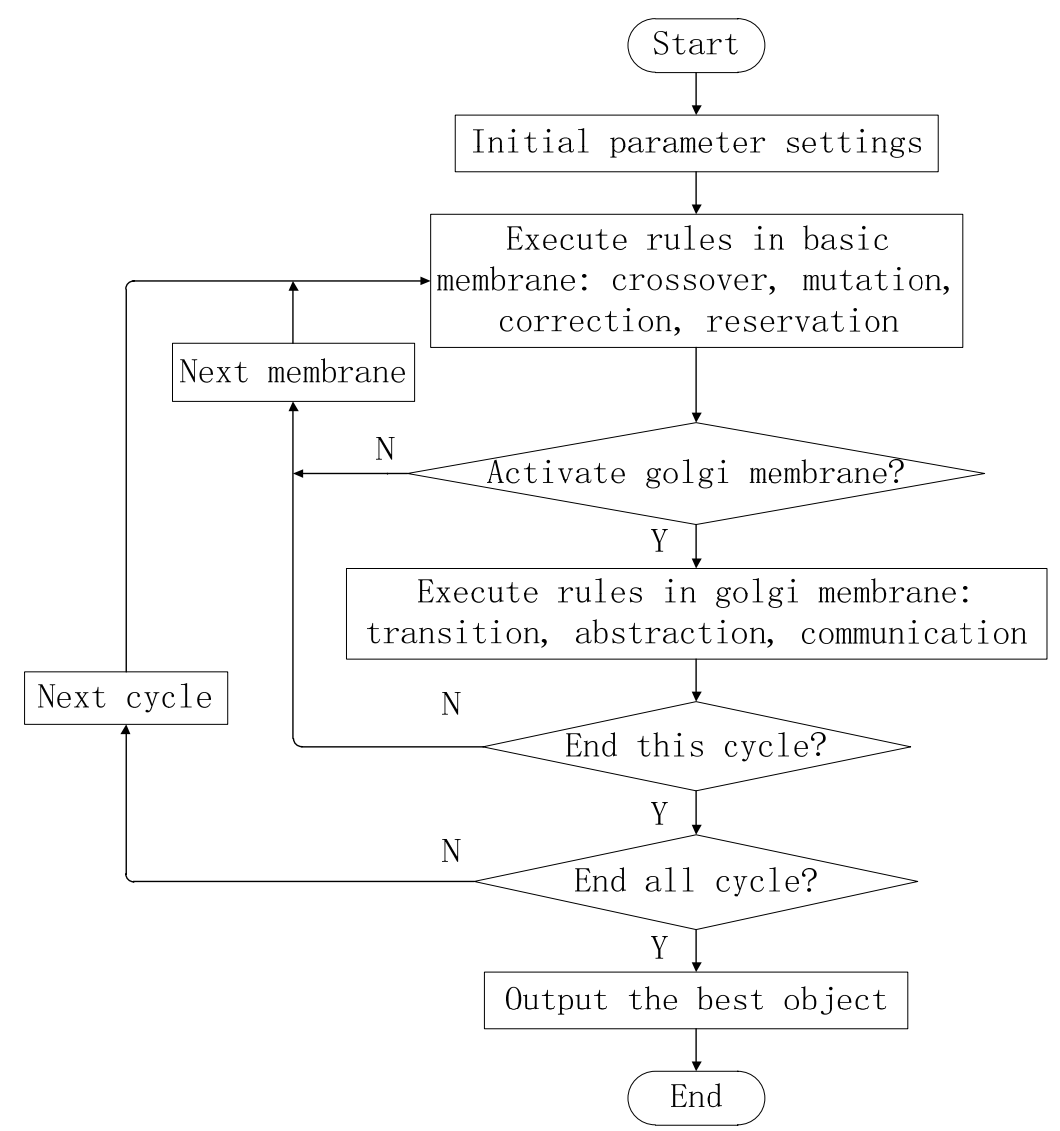

Figure 2 Flow chat of membrane computing algorithmic based dynamic economic dispatch.

\section{Simulation Results}

The IEEE 39-bus system is used as the test example, scheduling for 24 hours a day. The parameter settings of the proposed method are: the amount of repeated trials, $N_{r}=20$; the amount of computing cycle, $N_{c}=20$; the amount of parallel basic membranes, $N_{b}=20$; the amount of objects in basic membrane, $N_{o}=8$; the amount of communicating objects, $N_{c o}=2$; the amount of reserve objects, $N_{S V}=2$. The setting computational probability of crossover and mutation rules are 0.95 and 0.95. The golgi membrane is activated every three basic membrane after the second cycle, and the setting computational probability of transition and abstraction rules are 0.9 and 0.9 .

The results are listed in Table 1. It is obvious that the proposed method is more efficient comparing with GA and PSO: the best, mean, median, worst values and the standard deviation are all less than the other two methods, and the average computation time is shorter, indicating that the stability and the computational efficiency is both better than GA and PSO. 
Table 1 Comparison of different algorithms.

\begin{tabular}{ccccccc}
\hline Method & Best & Mean & Median & Worst & St. dev & Avg.t(s) \\
\hline GA & 675235 & 678653 & 679121 & 681103 & 1858.34 & 198.91 \\
PSO & 671242 & 676580 & 676964 & 680566 & 2305.68 & 115.86 \\
Proposed & 668461 & 670002 & 669900 & 671778 & 1014.95 & 41.75 \\
\hline
\end{tabular}

The convergence of the proposed method is shown in Figure 3. The best trial converges to a stable value around the 110th basic membranes, and the average result converges to a stable value around the 200th basic membranes, indicating the convergence and stability of the proposed method is good.

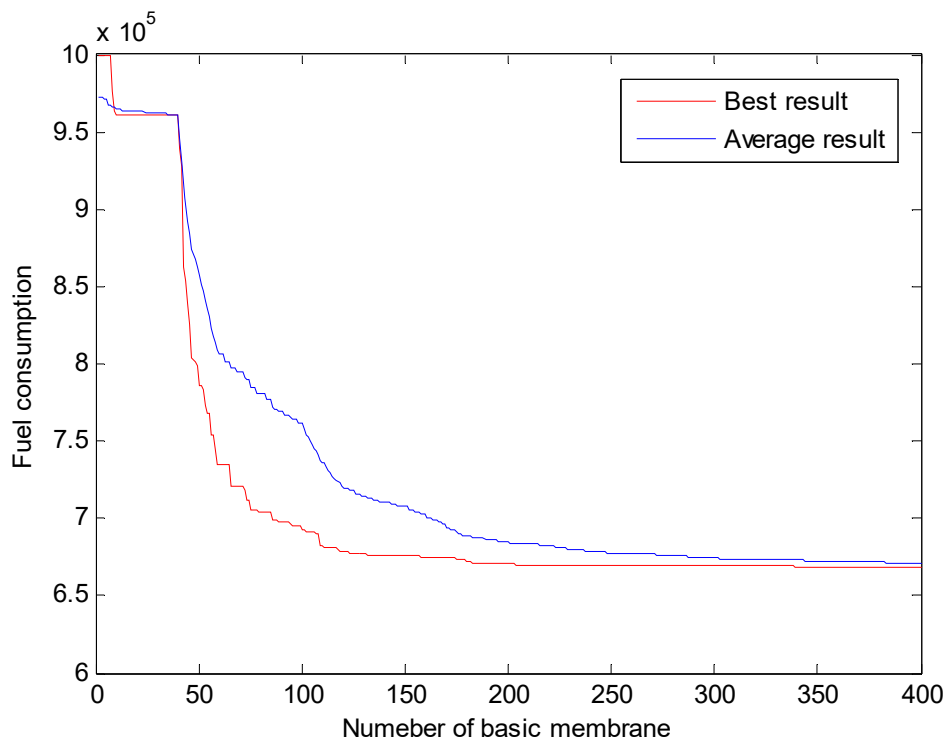

Figure 3 Convergence of the proposed method.

\section{Conclusions}

The membrane computing method is proposed to solve the power system dynamic economic dispatch problem in this paper. The method converges to a good value after a series of evolution rules executed. The efficiency and stability of the proposed method is proved by numerical example, which is superior to GA and PSO. The simulation results show that the optimization problem of dynamic economic dispatch can be solved effectively, thus the proposed method will be improved to solve the power system unit commitment problem in next research work.

\section{References}

[1] Păun G. Computing with membranes [J]. Journal of Computer and System Sciences, 2000, 61(1): 108-143.

[2] Zhang Gexiang. Membrane computing: Theory and Application [M] Beijing: Science Press, 2014.12.

[3] Zhang Gexiang, Pan Linqiang. A Survey of Membrane Computing As a New Branch of Natural Computing

[J]. Chinese Journal of Computers, 2010, 02:208-214.

[4] Lu Yu. Optimization Control in Unit Group Based on Decomposition-Coordination Method of Large Scale Systems [D]. North China Electric Power University (Beijing), North China Electric Power University, 2015. 
[5] Jian Ganyang, Liu Mingbo, Lin Shunjiang. Storage Modeling and Approximate Dynamic Programming Algorithm for Stochastic Dynamic Economic Dispatch Problems [J]. Proceedings of the CSEE, 2014, 34(25):4333-4340.

[6] Zhao Qingbo, Sun Lan, Guo Yan, etc. A Generation Resource Scheduling System Based on Lagrangian Relaxation Approach [J]. Automation of Electric Power Systems, 2004, 28(18):76-79.

[7] Wang Xin, Qin Fu, Yang Chunhua, etc. Short Term Environmental/Economic Generation Scheduling Based on Chaos Genetic Hybrid Optimization Algorithm [J]. Proceedings of the CSEE, 2006, 26(11):128-133.

[8] Liu Jianming, Li Maojun. Economic Dispatch of Hydro Power System Based on an Improved Genetic Algorithm [J]. Proceedings of the CSU-EPSA, 2007, 19(5):39-44.

[9] Liu Gang, Peng Chunhua, Xiang Longyang. Economic-Environmental Dispatch Using Improved MultiObjective Particle Swarm Optimization [J]. Power System Technology, 2011(7):139-144.

[10] Liu Yong, Hou Zhijian, Jiang Chuanwen. Economic Dispatch by a modified particle swarm Optimization Algorithm [J]. Power System Protection and Control, 2006, 34(20):24-27.

[11] Yang Tian. Cell Biology [M] Beijing: People's Medical Publishing House, 2010.8.

[12] Zhao J, Wang N. A bio-inspired algorithm based on membrane computing and its application to gasoline blending scheduling [J]. Computers \& Chemical Engineering, 2011, 35(2):272-283.

[13] Xiao J H, Huang Y F, Cheng Z. A Bio-Inspired Algorithm based on Membrane Computing for Engineering Design problem [J]. International Journal of Computer Science Issues, 2013, 10(1):580-588.

[14] Liu Chuang, Han Ming, Xing Jun. A global intelligent optimization algorithm based on membrane systems [J]. Chinese Journal of Electronics, 2013, 41(5):871-877.

[15] Huang L, Wang N. An Optimization Algorithm Inspired by Membrane Computing [J]. 2006, 4222:49-52.

[16] Huang Liang, He Xiongxiong, Wang Ning, et al. P systems based multi-objective optimization algorithm [J]. Progress in Natural Science: Materials International, 2007, 17(4):458-465. 